\title{
Hip Fracture Risk in Antiepileptic Drug Initiators and Non-Initiators with Alzheimer's Disease
}

\author{
Federica Pisa ${ }^{\mathrm{I}-3}$ \\ Jonas Reinold (D) ${ }^{1-3}$ \\ Piia Lavikainen (1D) 1,2 \\ Marjaana Koponen 1,2,4 \\ Heidi Taipale (iD) 1,2,5,6 \\ Antti Tanskanen (iD ${ }^{5-7}$ \\ Jari Tiihonen (D) 5,6 \\ Sirpa Hartikainen ${ }^{1,2}$ \\ Anna-Maija Tolppanen (D) 1,2 \\ 'School of Pharmacy, University of Eastern \\ Finland, Kuopio, Finland; ${ }^{2}$ Kuopio Research \\ Centre of Geriatric Care, University of \\ Eastern Finland, Kuopio, Finland; \\ ${ }^{3}$ Department of Clinical Epidemiology, \\ Leibniz Institute for Prevention Research \\ and Epidemiology - BIPS, Bremen, \\ Germany; ${ }^{4}$ Centre for Medicine Use and \\ Safety, Faculty of Pharmacy and \\ Pharmaceutical Sciences, Monash \\ University, Melbourne, Victoria, Australia; \\ ${ }^{5}$ Department of Clinical Neuroscience, \\ Karolinska Institutet, Stockholm, Sweden; \\ ${ }^{6}$ Department of Forensic Psychiatry, \\ Niuvanniemi Hospital, University of Eastern \\ Finland, Kuopio, Finland; ${ }^{7}$ Impact \\ Assessment Unit, National Institute for \\ Health and Welfare, Helsinki, Finland
}

Correspondence: Federica Pisa Bayer, Müllerstrasse 178, Berlin, 13353,

Germany

Email federica.pisa@bayer.com
Objective: To determine the risk of hip fracture in persons with Alzheimer's disease (AD) who initiated antiepileptic drugs (AEDs).

Methods: In the Medication use and AD (MEDALZ) cohort of 70,719 Finnish community dwellers with clinically verified incident AD diagnosis in 2005-2011, we identified all incident users of AEDs using national Prescription register. AEDs were classified as older (valproate, carbamazepine, clonazepam, phenytoin, levetiracetam, primidone) or newer (pregabalin, gabapentin, oxcarbazepine, lamotrigine, topiramate). We matched each user to 2 non-users. Incident hip fractures until 2015 were identified from the Care register for health care. We calculated inverse probability of treatment weighted hazard ratios (HR), with $95 \%$ confidence intervals, using Cox regression.

Results: Altogether 5522 incident users were identified and matched to 11,044 non-users (in both groups, women: $65 \%$; median age: 81 years). Altogether $53.3 \%$ of users initiated with newer AEDs (pregabalin 79.8\%, gabapentin 10.2\%) while 46.7\% initiated with older AEDs (valproate $67.6 \%$, carbamazepine $13.0 \%$ ). Age- and sex-adjusted IR of hip fracture per 100 person-years was $1.8(95 \%$ CI $1.6-1.9)$ in non-users and 2.0 (95\% CI 1.8-2.2) in users. Increased risk of hip fracture was observed in users (HR 1.17, 95\% CI 1.05-1.30) compared with non-users. The risk was higher for short duration of use $(<14$ weeks, HR $3.64,95 \%$ CI $2.90-4.58$ ) than for medium duration (14 to $<64$ weeks, HR $1.74,95 \%$ CI $1.48-2.05$ ) or $\geq 64$ weeks' use (HR 1.23, 95\% CI 1.08-1.40), compared to non-users with same follow-up time. Older AEDs had HR of 1.46 (1.03-2.08) compared with newer AEDs. Conclusion: Our results imply that AED use is associated with an increased risk of hip fracture in people with $\mathrm{AD}$. These findings prompt careful consideration before prescribing AEDs to persons with AD. Persons with AD treated with antiepileptics should be carefully monitored due to their increased risk of falling and fractures.

Keywords: antiepileptic drugs, hip fracture, Alzheimer's disease, pharmacoepidemiology

\section{Introduction}

Antiepileptic drugs (AEDs) have been consistently associated with an increased risk of fractures, including hip fractures. ${ }^{1-4}$ In a meta-analysis of observational studies, the risk of hip fracture was almost doubled in users of AEDs compared with non-users. ${ }^{5}$ However, most studies have examined young or mid-aged adults with epilepsy, while very little evidence exists for older adults treated for indications other than epilepsy. The use of antiepileptics, especially the newer antiepileptics such as pregabalin and gabapentin, on other indications has become more common. In persons with AD, AEDs are mainly used for central neuropathic pain, ${ }^{6}$ and neuropsychiatric symptoms of cognitive disorders (eg, agitation and 
aggression $){ }^{7}$ although, particularly in the case of neuropathic pain, this goes against recent recommendations due to an increased risk of falls. ${ }^{8}$ Moreover, in a recent systematic review of randomized controlled trials, valproate showed no benefit in treating dementia-related agitation and a high rate of adverse effects, including sedation was observed among valproate users. ${ }^{9}$ Still, the risk-benefit profile of most AEDs in persons with $\mathrm{AD}$ is still largely unclear $^{10-13}$ and their adverse effects, including effects on cognition are of concern, given that cognitive status is already impaired due to $\mathrm{AD}$ itself.

A screening study for safety signals in a large Finnish cohort of persons with $\mathrm{AD},{ }^{14}$ identified an increased risk of hip fracture associated with pregabalin and valproate. In this cohort, ${ }^{15}$ use of AEDs after AD diagnosis increased from about $4 \%$ to about $8 \%$ within 5 years and the most common AEDs were new agents, such as pregabalin and gabapentin. Therefore, it is very important to confirm and further investigate the relation of AEDs with hip fracture in persons with $\mathrm{AD}$, who are a well-known high-risk group for hip fracture. ${ }^{16}$ Hip fractures are indeed a major health problem in older adults, ${ }^{17}$ and strongly affect their health and well-being, substantially increasing morbidity, ${ }^{18}$ shortterm mortality, ${ }^{19-22}$ and reducing autonomy and quality of life. ${ }^{23,24}$ As a result, health-care costs are also substantially increased. ${ }^{25-28}$

In this study, we compare the risk of hip fracture between AED initiators and non-initiators with $\mathrm{AD}$ in a nationwide Finnish cohort of persons with AD.

\section{Methods}

\section{Study Population and Data Sources}

The study population was obtained from the Medication and Alzheimer's disease (MEDALZ) cohort, which has been described in detail elsewhere. ${ }^{29}$ Briefly, the MEDALZ cohort includes community-dwelling residents of Finland who received a clinically verified diagnosis of AD from 2005 to 2011 (N=70,719) (Figure 1). Persons with diagnosis of AD were identified from the Special Reimbursement register (SRR). This register and the other sources of data are described in Table e-1. To be registered in the SRR with a diagnosis of $\mathrm{AD}$, a person has to fulfil the following clinical criteria: he/she (1) had symptoms consistent with $\mathrm{AD}$, (2) experienced a decrease in social capacity over a period of at least 3 months, (3) underwent a computed tomography/magnetic resonance imaging scan, (4) had possible alternative diagnoses excluded, and (5) had a diagnosis of AD made by a registered geriatrician or neurologist. The diagnosis of $\mathrm{AD}$ is based on the National Institute of Neurological and Communicative Disorders and Stroke and the Alzheimer's Disease and Related Disorders Association ${ }^{30}$ and Diagnostic and Statistical Manual of Mental Disorders, Fourth Edition, criteria for AD. ${ }^{31}$

For each member of the cohort, information on drug use, diagnoses, hospitalizations and institutionalizations were extracted from nationwide registers (Table e-1 and Figure e-1). Data on dispensed drugs were extracted from the Prescription register (years 1995-2015), on hospitalizations (including discharge diagnoses coded according to the 10th revision of the WHO International Classification of Diseases and Related Health Problems (ICD-10) from the Hospital Discharge register (1972-2015); on selected chronic diseases diagnoses from the SRR (1972-2015). Moreover, data on institutionalization (including end and start date) were obtained from the Social Insurance Institution of Finland (1972-2015), data on deaths (2005-2015) and socioeconomic status (1972-2015) from Statistics Finland.

In the MEDALZ cohort, we identified all persons who initiated AEDs after AD diagnosis (incident users) and matched each incident user to 2 non-users of AEDs.

\section{Incident Users of AEDs}

Incident users were defined as persons who had a first dispensation of an AED after AD diagnosis without having filled one within 1 year before. We restricted the study to incident users to avoid bias related to the depletion of susceptible prevalent users and under-ascertainment of earlier events. ${ }^{32}$

For incident users, the date of the first dispensation of an AED after AD diagnosis was defined as the index date. Duration of use was calculated starting from the index date until censoring (ie, until discontinuation of AED use, switch to or addition of an AED of the other group, death, start of continuous hospitalization/institutionalization lasting $\geq 90$ days, end of the study (December 31, 2015), and is thus independent of timing of hip fracture.

AED dispensations were identified in the Prescription register through the ATC code N03A. Individual drugs were classified as older AEDs (valproate N03AG01, carbamazepine N03AF01, clonazepam N03AE01, phenytoin $\mathrm{N} 03 \mathrm{AB} 02$, levetiracetam N03AX14, primidone N03AA03) and newer AEDs (pregabalin N03AX16, gabapentin N03AX12, oxcarbazepine N03AF02, lamotrigine N03AX09, and topiramate N03AX11). 


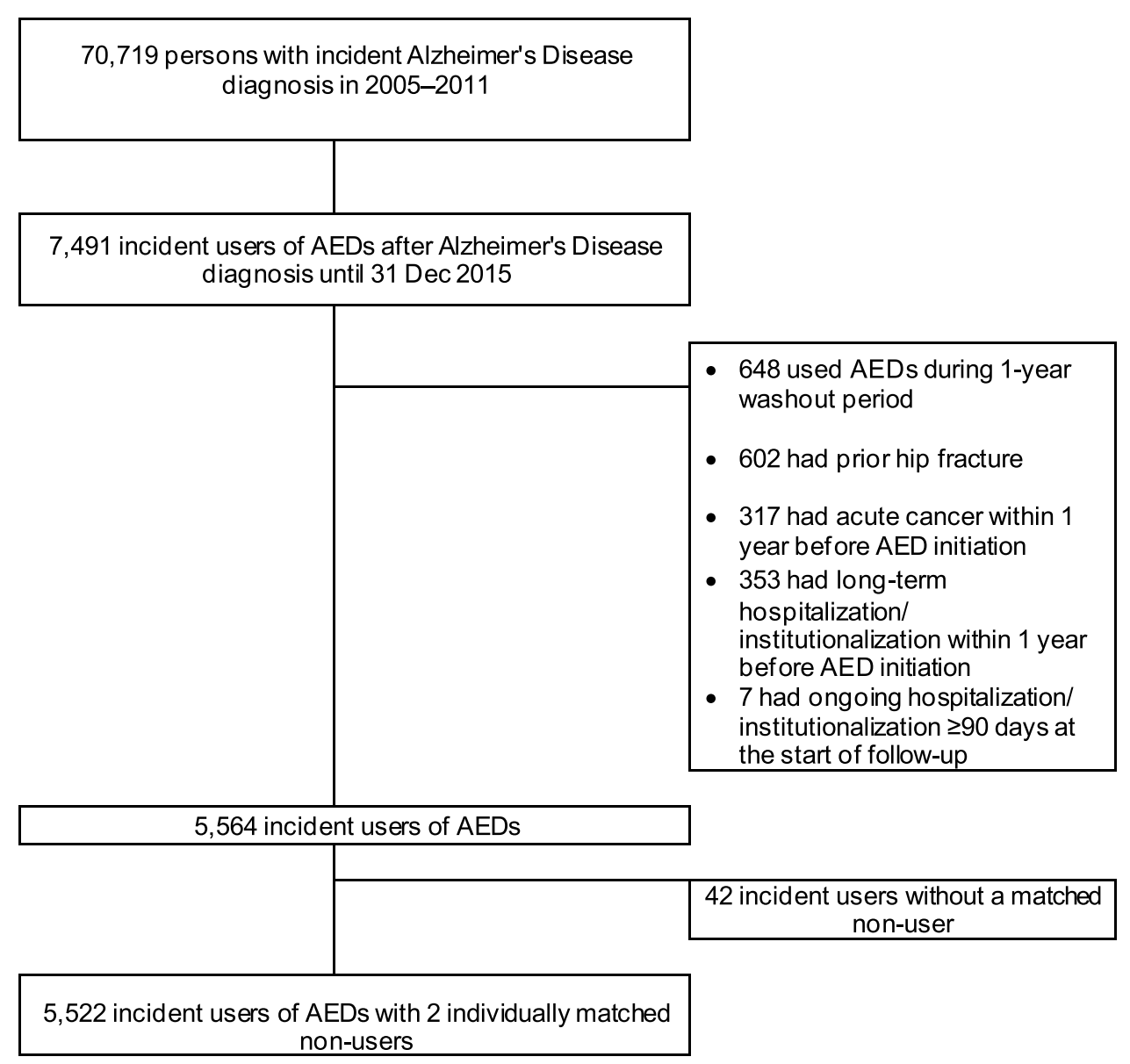

Figure I Flow chart describing the study population.

We excluded incident users who experienced hip fracture any time between 1972 and index date, those who had an acute cancer at index date, and those who were hospitalized or institutionalized for $>182$ days during 1 year before or who were currently hospitalized for $\geq 90$ days at entry date. We excluded persons with long-term hospitalization or institutionalization because drug exposure cannot be accurately measured during in-hospital stays. Definitions used for exclusion criteria are displayed in Table e-1.

Using the PREscriptions to Drug Use Periods PRE2DUP method, ${ }^{33}$ dispensation purchase data of AEDs have been transformed to drug use periods. This method constructs exposure periods and estimates the dose used during the period by considering the purchased amount in defined daily doses, recorded in the prescription register. This method models personal purchase pattern for each ATC code and considers stays in hospital and long- term care facilities (during which drug use is not recorded in the prescription register), possible stockpiling of drugs, and package information. Drug use based on PREDUP has been compared with self-reported drug use, ${ }^{34}$ and findings showed a very high agreement for central nervous system drugs.

As PREDUP modelling is based on the individual ATC code, duration of any AED use was obtained combining overlapping drug use periods of AEDs. Thus, during any AED use, a person may change AED.

\section{Matched Non-Users}

For each incident user, two non-users of AEDs were matched by sex, age ( \pm 1 year), and time since AD diagnosis ( \pm 183 days) using incidence density sampling without replacement. For non-users, the matching date was defined as the index date. Non-users had to be alive and not hospitalized at the cohort entry date of the correspond- 
ing incident user. We applied to non-users the same exclusion criteria applied to incident users.

\section{Follow-Up}

Follow-up started on the index date (date of the first dispensation of an AED after AD diagnosis for incident users and the matched date for non-users).

In the analysis comparing incident users with non-users, each person was followed-up from index date to the date of incident hip fracture, death, start of continuous hospitalization/institutionalization lasting $\geq 90$ days, end of the study (December 31, 2015), AED use discontinuation (for users), or AED initiation (for non-users), whichever occurred first. In the analysis comparing incident users of older and newer AEDs, the follow-up ended on the date of switch to or addition of an AED of the other group in addition to the other censoring criteria (date of incident hip fracture, death, start of continuous hospitalization/institutionalization lasting $\geq 90$ days, or end of the study), whichever occurred first.

\section{Outcome}

Within the person-time of follow-up, we identified all persons who experienced an incident hip fracture, defined as (1) the first hospitalization with ICD-10 code for fracture of neck of femur (S72.0), pertrochanteric fracture (S72.1), or subtrochanteric fracture (S72.2), or (2) death with the same ICD-10 codes for causes.

\section{Covariates}

We ascertained at baseline characteristics that are risk factors for hip fracture and/or are associated with use of AEDs, including co-morbidities and use of drugs other than AEDs. The definitions and classifications used to measure these characteristics are described in Table e-2. Briefly, co-morbidities have been identified mainly through corresponding ICD codes in the Hospital Discharge register. Some co-morbidities were identified using additional data, for instance, dispensations of specific drugs (eg, osteoporosis was identified through hospitalization codes for osteoporosis- ICD-10 M80 and M81and/or dispensations of osteoporosis drugs - M05BA, $\mathrm{M} 05 \mathrm{BB}, \mathrm{M} 05 \mathrm{BC}$, and M05BX any time before cohort entry) or special reimbursement for chronic diseases (eg, rheumatoid arthritis was identified through hospitalization codes - ICD-10 M05, M06, M45 - and/or the special reimbursement code for this disease).
Use of drugs other than AEDs at baseline was ascertained within 12 months prior to cohort entry based on dispensations with specific ATC codes in the Prescription register.

Socioeconomic status was defined based on the highest occupational social class recorded for a person from 1972 up to 3 years prior to the AD diagnosis and it was classified into four groups by Statistics Finland. These groups corresponded to low (unemployed and students), medium (employees and lower clerical workers) and high (higher clerical workers, professionals and entrepreneurs) status. A fourth group included persons with unknown socioeconomic status or missing information.

\section{Statistical Analysis}

Age- and sex-adjusted incidence rates (IRs), with 95\% confidence intervals ( $95 \%$ CIs), of hip fracture have been calculated using Poisson regression and expressed as number of incident cases per 100 person-years. IRs have been calculated separately in incident users and non-users as well as in incident users of older and newer AEDs.

Unadjusted and adjusted hazard ratios (HR), with 95\% confidence intervals $(95 \% \mathrm{CI})$, were estimated using Cox proportional hazard regression. The proportional hazards assumption was assessed using visual examination of hazard functions and graphic and goodness-of-fit testing using Schoenfeld residuals.

To balance the compared groups regarding baseline covariates, we applied Inverse Probability of Treatment Weighting (IPTW). ${ }^{35}$ We conducted two sensitivity analyses, one using propensity score for covariate adjustment and the other using stabilised IPTW.

We used conditional logistic regression to estimate the propensity score as the probability of receiving any AED vs none conditioned to baseline covariates. All baseline covariates (Table e-2) were included in the propensity score. IPTW was calculated in incident users as 1-propensity score and in non-users as 1/(1-propensity score). ${ }^{36}$ To quantitatively assess the degree to which IPTW weighting had removed systematic differences at baseline between incident users and non-users, we calculated unweighted and IPTW weighted standardized mean differences (SMDs) between incident users and non-users for each baseline covariate. ${ }^{37} \mathrm{New}$ propensity scores (and, thus, IPTWs) were derived for the older vs newer AED comparison analyses based on the probability of receiving newer AED vs older AED conditional on baseline covariates.

To avoid immortal time bias in analyses assessing the risk of hip fracture per AED use duration, non-users with 
the same follow-up duration were used as reference category in the use/non-use analyses. In the duration-wise analyses of older vs newer AEDs, the comparisons were performed between users of older and newer AEDs with the same duration of use.

All analyses were performed using SAS statistical software, version 9.3 (SAS InstituteC, Inc., Cary, NC).

\section{Standard Protocol Approvals, Registrations, and Patient Consents}

The MEDALZ study was approved by the register maintainers. According to Finnish legislation, ethics committee approval or informed consent were not required for this study because only pseudonymised register-based data were used, the study participants were not contacted and treatment was not affected by participation in the study. Data were pseudonymised before submission to the researchers.

\section{Data Availability Statement}

The data used to conduct the research are available from the corresponding author but restrictions by the register maintainers and Finnish legislation apply to the availability of these data. Therefore, the data are not publicly available without permissions of the register maintainers.

\section{Results}

A total of 5564 incident users of any AED were identified and $5522(99.2 \%)$ of them were matched to 11,044 nonusers; 42 incident users without matching non-user were excluded from further analysis (Figure 1). In both groups, about $65 \%$ were women and median age was 81 years (Table 1). At baseline, users had co-morbidities and used drugs more frequently than non-users. The largest differences were observed for opioids, benzodiazepines and related drugs, and epilepsy. These differences were balanced after IPTW, as indicated by SMD $<10 \%$ between users and non-users. Distributions of stabilized IPTWs between users and non-users were comparable, with significant overlap (mean weight in users 1.00, range 0.73-7.08, mean weight in non-users 0.99 , range $0.35-4.52$ ).

Among incident users, 2945 (53.3\%) used newer AEDs and 2577 (46.7\%) used older AEDs (Table 2). The most commonly used newer AEDs were pregabalin (79.8\%), gabapentin (10.2\%), and oxcarbazepine (8.7\%); the most commonly used older AEDs were valproic acid (67.6\%), carbamazepine (13.0\%), and clonazepam (10.2). Compared to users of newer AEDs, users of older AEDs were more frequently men, had shorter median time since diagnosis (656 vs 995 days), had more frequently epilepsy, and used more frequently memantine and antipsychotics (Table 2). Conversely, other co-morbidities and drugs were more common in users of newer AEDs, such as osteoporosis, vision disturbances, and opioids. The SMD exceeded 10\% for 19 co-morbidities and drugs at baseline, with differences between $30 \%$ and $50 \%$ for opioids, antipsychotics, epilepsy, NSAIDs, and between $20 \%$ and $30 \%$ for PPIs, memantine, vision disturbances, and osteoporosis. These differences were balanced after IPTW, as indicated by SMDs $<10 \%$ between users of newer and older AEDs for all except epilepsy (SMD 11.2\%).

During follow-up, 673 (6.1\%) non-users and 355 (6.4\%) users experienced an incident hip fracture, with age-sex-adjusted IR (95\% CI) of 1.8 (1.6-1.9) and 2.0 (1.8-2.2) per 100 person-years, respectively (Table 3 ). The IR was higher in users of older AEDs (2.6; 2.1-3.3) than in users of newer AEDs (1.4; 1.1-1.9).

Incident users of AEDs had HR slightly higher than nonusers (IPTW HR 1.17; 1.05-1.30) (Table 3). Among incident users, the IPTW HR was $3.64(2.90-4.58)$ in those with short duration of use (<14 weeks), 1.74 (1.48-2.05) in those with medium duration (14 to $<64$ weeks) and $1.23(1.08-1.40)$ in those who used AEDs for $\geq 64$ weeks in comparison to nonusers with same follow-up time. The results from the sensitivity analyses using stabilized weights and propensity score adjustment had larger confidence intervals but were comparable to the main analyses.

The risk was not modified by age ( $p$ for interaction between AED use and age $=0.63$ ), but larger HR's were observed among younger users (age $<65$ years) than older users (Figure 2).

The risk of hip fracture was higher in users of older (IPTW HR 1.46; 1.03-2.08) compared to users of newer AEDs. In users of older AEDs, the relative risk was $1.27(0.83-1.95)$ in those with short duration of use, 2.00 (1.33-3-01) in those with medium and 1.06 (0.75-1.49) in those with long duration of use compared to users of new AEDs with same follow-up time. The results from the sensitivity analyses with stabilized weights or propensity score adjustment were in line with results of the main analyses, although in both sensitivity analyses the point estimates for short and medium duration were stronger, and those for old vs new comparison weaker than in the main analyses. 
Table I Characteristics of Incident Users of AEDs and Matched Non-Users with AD and Standardized Mean Differences

\begin{tabular}{|c|c|c|c|c|c|c|}
\hline & \multicolumn{2}{|c|}{ Users $(\mathrm{N}=5522)$} & \multicolumn{2}{|c|}{ Non-Users $(\mathrm{N}=1 \mathrm{I}, 044)$} & \multicolumn{2}{|c|}{ Standardized Mean Difference (\%) } \\
\hline & $\mathbf{N}$ & $\%$ & $\mathbf{N}$ & $\%$ & Unweighted & After IPTW \\
\hline \multicolumn{7}{|l|}{ Age (years) ${ }^{a}$} \\
\hline$<64$ & 431 & 7.8 & 1392 & 12.6 & 15.9 & 1.8 \\
\hline $65-74$ & 1360 & 24.6 & 3663 & 33.2 & & \\
\hline $75-84$ & 2909 & 52.7 & 5005 & 45.3 & & \\
\hline $85+$ & 822 & 14.9 & 984 & 8.9 & & \\
\hline Median (25;75 percentile) & \multicolumn{2}{|c|}{$81.1(75.8 ; 85.5)$} & \multicolumn{2}{|c|}{$81.0(75.6 ; 85.2)$} & - & - \\
\hline \multicolumn{7}{|l|}{ Sex } \\
\hline Men & 1941 & 35.2 & 3882 & 35.2 & 0.0 & 0.5 \\
\hline Women & 3581 & 64.9 & 7162 & 64.9 & & - \\
\hline \multicolumn{7}{|l|}{ 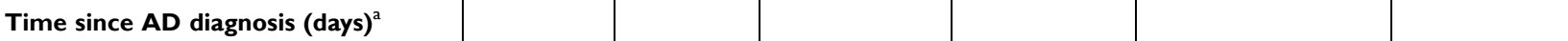 } \\
\hline$<315$ days & 1373 & 24.9 & 2572 & 23.3 & 3.7 & 1.1 \\
\hline 315 to $<803$ & 1382 & 25.0 & 2727 & 24.7 & 0.8 & 0.8 \\
\hline 803 to $<1465$ & 1385 & 25.1 & 2759 & 25.0 & 0.2 & 0.4 \\
\hline I 465 and above & 1382 & 25.0 & 2986 & 27.0 & 4.6 & 2.3 \\
\hline Median (25;75 percentile) & \multicolumn{2}{|c|}{$803(3 \mid 5 ; 1465)$} & \multicolumn{2}{|c|}{$850(344 ; 1533)$} & & \\
\hline \multicolumn{7}{|l|}{ Duration of follow-up (days) } \\
\hline Median (25;75 percentile) & \multicolumn{2}{|c|}{$810.5(293 ; 1504)$} & \multicolumn{2}{|c|}{$973(442 ; 1617)$} & & \\
\hline \multicolumn{7}{|l|}{ Health condition at baseline } \\
\hline \multicolumn{7}{|l|}{ Psychiatric and neurological } \\
\hline Epilepsy & 542 & 9.8 & 304 & 2.8 & 27.4 & 0.4 \\
\hline Depression & 463 & 8.4 & 645 & 5.8 & 10.0 & 0.4 \\
\hline Schizophrenia & 157 & 2.8 & 284 & 2.6 & 1.7 & 0.6 \\
\hline \multicolumn{7}{|l|}{ Hip fracture-related } \\
\hline Vision disturbances ${ }^{\mathrm{b}}$ & 2104 & 38.1 & 3181 & 28.8 & 19.2 & 0.3 \\
\hline Osteoporosis $^{c}$ & 1036 & 18.8 & 1479 & 13.4 & 14.1 & 0.5 \\
\hline Any fracture & 797 & 14.4 & $|24|$ & 11.2 & 9.2 & 0.1 \\
\hline Head trauma & 439 & 8.0 & 687 & 6.2 & 6.0 & 1.0 \\
\hline Rheumatoid arthritis $^{d}$ & 266 & 4.8 & 496 & 4.5 & 1.5 & 0.3 \\
\hline Alcohol abuse & 186 & 3.4 & 332 & 3.0 & 1.4 & I.I \\
\hline \multicolumn{7}{|l|}{ Other conditions } \\
\hline Cardiovascular diseases ${ }^{\mathrm{e}}$ & 3595 & 65.1 & 6179 & 56.0 & 18.2 & 0.2 \\
\hline Diabetes $^{d}$ & 1296 & 23.5 & 2298 & 20.8 & 6.5 & 0.3 \\
\hline Stroke & 847 & 15.3 & 1143 & 10.4 & 14.6 & 0.8 \\
\hline Asthma ${ }^{d}$ & 622 & 11.3 & 1055 & 10.0 & 5.6 & $<0.1$ \\
\hline History of any cancer & 488 & 8.8 & 1110 & 10.1 & 4.1 & $<0.1$ \\
\hline Chronic renal disease & 85 & 1.5 & 86 & 0.8 & 7.1 & 0.4 \\
\hline Chronic liver disease & 27 & 0.5 & 82 & 0.7 & 2.9 & 0.9 \\
\hline \multicolumn{7}{|l|}{ Drugs at baseline } \\
\hline \multicolumn{7}{|l|}{ CNS drugs } \\
\hline Benzodiazepines and related drugs & 2338 & 42.3 & 2859 & 25.9 & 33.1 & 0.5 \\
\hline Antidepressants & 2307 & 41.8 & 3289 & 29.8 & 23.6 & 0.6 \\
\hline Memantine & 2257 & 40.9 & 3970 & 36.0 & 6.2 & 0.3 \\
\hline Antipsychotics & 1963 & 35.6 & 2698 & 24.4 & 21.0 & 1.1 \\
\hline Opioids & 1265 & 22.9 & 1007 & 9.1 & 36.5 & 0.5 \\
\hline Antiparkinsonians & 297 & 5.4 & 510 & 4.6 & 3.4 & 0.1 \\
\hline
\end{tabular}

(Continued) 
Table I (Continued).

\begin{tabular}{|c|c|c|c|c|c|c|}
\hline & \multicolumn{2}{|c|}{ Users $(\mathrm{N}=5522)$} & \multicolumn{2}{|c|}{ Non-Users $(\mathrm{N}=\mathrm{I} \mid \mathrm{l}, 044)$} & \multicolumn{2}{|c|}{ Standardized Mean Difference (\%) } \\
\hline & $\mathbf{N}$ & $\%$ & $\mathbf{N}$ & $\%$ & Unweighted & After IPTW \\
\hline \multicolumn{7}{|l|}{ Other drugs } \\
\hline Cardiovascular drugs $\mathrm{f}$ & 4535 & 82.1 & 8379 & 75.9 & 15.1 & 0.3 \\
\hline PPls & 1811 & 32.8 & 2299 & 20.8 & 26.7 & $<0.1$ \\
\hline NSAIDs & 1224 & 22.2 & 1597 & 14.5 & 19.2 & 0.1 \\
\hline Corticosteroids, systemic & 485 & 8.8 & 633 & 5.7 & 11.3 & 0.6 \\
\hline Estrogens & 481 & 8.7 & 760 & 6.9 & 6.5 & 0.2 \\
\hline Drugs for urinary incontinence & 313 & 5.7 & 551 & 5.0 & 3.7 & $<0.1$ \\
\hline \multicolumn{7}{|l|}{ Socioeconomic status } \\
\hline Highest & 2798 & 50.7 & 4241 & 38.4 & 5.8 & 0.6 \\
\hline Middle & 980 & 17.8 & 2187 & 19.8 & 0.2 & 0.1 \\
\hline Lowest & 942 & 17.1 & 2606 & 23.6 & 3.0 & 0.2 \\
\hline Unknown & 802 & 14.5 & 2010 & 18.2 & 3.8 & 1.1 \\
\hline
\end{tabular}

Notes: a Matching variables. ${ }^{\mathrm{b}}$ Includes hospital diagnosis of cataract, macular degeneration, and glaucoma. ${ }^{\mathrm{c} C o m p o s i t e}$ of discharge diagnosis (code M80 and M8I) and dispensation data (bisphosphonates M05BA and M05BB, bone morphogenetic proteins M05BC, other drugs affecting bone structure and mineralization M05BX (eg, strontium ranelate, denosumab)). ${ }^{\mathrm{d}}$ Composite variables based on both hospitalization and special reimbursement data. ${ }^{\mathrm{e}}$ It includes hypertension, heart failure, peripheral arterial disease, atrial fibrillation, coronary heart disease. IIt includes cardiac glycosides (COIAA), antiarrhythmics (COIB), organic nitrates (C0IDA), diuretics (C03), beta blocking agents (C07), calcium channel blockers (C08), agents acting on the renin-angiotensin system (C09), lipid modifying agents ( $\mathrm{CIOA}, \mathrm{ClOBA})$, antithrombotic agents (B0IA).

Abbreviations: AD, Alzheimer's disease; AED, antiepileptic drug; CNS, central nervous system; HF, hip fracture; IPTW, inverse probability of treatment; NSAID, nonsteroidal anti-inflammatory drugs; PPI, proton pump inhibitors.

\section{Discussion}

In this cohort of persons with clinically confirmed $\mathrm{AD}$, there was a $17 \%$ increase in the risk of hip fractures in incident users of AEDs compared with non-users. However, when duration of use was considered, higher risk increase was observed for shorter term of use (less than 14 weeks) than for longer term use. In addition, users of older AEDs had a 46\% increased relative risk of hip fracture compared with users of newer AEDs.

To our knowledge, this is the first study on the risk of hip fractures associated with AEDs in persons with AD. Prior studies focused on young-adult persons with epilepsy, who are commonly treated for long periods and often in polytherapy. The increase in risk in our study has a lower magnitude than in prior studies showing a doubled risk of hip fractures associated with AEDs. ${ }^{5}$ This difference in magnitude may be explained by differences in study population regarding age and morbidity profile, as well as in indication, type of AEDs, and pattern of use. Firstly, persons with $\mathrm{AD}$ have a high background risk of hip fractures; therefore, the excess risk due to AEDs may be lower than in persons without cognitive disorders. Secondly, in persons with AD, AEDs are mostly used for neuropathic pain ${ }^{6}$ and to manage neuropsychiatric symptoms (eg, agitation and aggression). Consistently, in our cohort pregabalin and gabapentin were among the most commonly used AEDs, suggesting that indeed neuropathic pain and management of neuropsychiatric symptoms were the most probable indications.

The finding of a higher risk in persons with short duration of use is consistent with early adverse effects of AEDs (such as sedation, confusion, blurred vision, and ataxia) that occur mostly in the early stages of the treatment and increase the susceptibility to falls. ${ }^{38}$ Indeed, falls are the leading cause of fractures of the hip in older adults. ${ }^{39,40}$ However, the higher risk persisted also for those with medium duration of use (from 14 to $<64$ weeks) and longer term of use (64 weeks or longer), suggesting that early adverse events are likely not the only explanation. Indeed, sedation may also persist for longer period of time, not just in the beginning of use. Moreover, dose-related adverse effects may occur beyond early use, as when the dose is slowly and gradually increased as it should be done in vulnerable older persons to identify possible adverse effect. This is the case of hyponatremia, a risk factor for falling in elderly persons. $^{41}$

This study faces methodological challenges related to the observational design and use of healthcare databases. 
Table 2 Characteristics of Incident Users of Newer and Older AEDs with AD and Mean Standardized Differences

\begin{tabular}{|c|c|c|c|c|c|c|}
\hline & \multicolumn{4}{|c|}{ Users $^{a}$} & \multicolumn{2}{|c|}{ Standardized Mean Difference (\%) } \\
\hline & \multicolumn{2}{|c|}{ Newer AEDs ( $\mathbf{N = 2 9 4 5 )}$} & \multicolumn{2}{|c|}{ Older AEDs ( $N=2577)$} & \multirow[b]{2}{*}{ Unweighted } & \multirow[b]{2}{*}{ After IPTW } \\
\hline & $\mathbf{N}$ & $\%$ & $\mathbf{N}$ & $\%$ & & \\
\hline \multicolumn{7}{|l|}{ Age (years) } \\
\hline$<64$ & 132 & 4.5 & 299 & 11.6 & 26.4 & 5.4 \\
\hline $65-74$ & 625 & 21.2 & 735 & 28.5 & 26.4 & 5.4 \\
\hline $75-84$ & 1678 & 57.0 & $|23|$ & 47.8 & 26.4 & 5.4 \\
\hline $85+$ & 510 & 17.3 & 312 & 12.1 & 26.4 & 5.4 \\
\hline \multicolumn{7}{|l|}{ Median ( $25 ; 75$ percentile) } \\
\hline \multicolumn{7}{|l|}{ Sex } \\
\hline Men & 915 & 31.1 & 1026 & 39.8 & 18.4 & 2.5 \\
\hline Women & 2030 & 68.9 & $|55|$ & 60.2 & & \\
\hline \multicolumn{7}{|c|}{ Time since AD diagnosis (days) } \\
\hline$<315$ days & 840 & 28.5 & 533 & 20.7 & 18.3 & 0.3 \\
\hline 315 to $<803$ & 828 & 28.1 & 554 & 21.5 & 15.4 & 0.2 \\
\hline 803 to $<1465$ & 716 & 24.3 & 669 & 26.0 & 3.8 & 0.7 \\
\hline I 465 and above & 561 & 19.1 & 821 & 31.9 & 29.7 & 1.2 \\
\hline Median (25;75 percentile) & \multicolumn{2}{|c|}{$656(265 ; 1269)$} & \multicolumn{2}{|c|}{$995(402 ; 1697)$} & & \\
\hline \multicolumn{7}{|l|}{ Duration of follow-up (days) } \\
\hline Median (25;75 percentile) & \multicolumn{2}{|c|}{$982.00(437.00 ; 1660.00)$} & \multicolumn{2}{|c|}{$578.00(198.00 ; 1221.00)$} & & \\
\hline \multicolumn{7}{|c|}{ AED at start treatment (ATC) } \\
\hline Pregabalin (N03AXI6) & 2349 & 79.8 & - & & - & - \\
\hline Gabapentin (N03AXI2) & 300 & 10.2 & - & & - & - \\
\hline Oxcarbazepine (N03AF02) & 255 & 8.7 & - & & - & - \\
\hline Lamotrigine (N03AX09) & 31 & 1.1 & - & & - & - \\
\hline Topiramate (N03AXII) & 9 & 0.3 & - & & - & - \\
\hline Valproic acid (N03AG0I) & - & & 1742 & 67.6 & - & - \\
\hline Carbamazepine (N03AFOI) & - & & 334 & 13.1 & - & - \\
\hline Clonazepam (N03AEOI) & - & & 263 & 10.2 & - & - \\
\hline Phenytoin (N03AB02) & - & & 119 & 4.6 & - & - \\
\hline Levetiracetam (N03AXI4) & - & & 79 & 3.1 & - & - \\
\hline Primidone (N03AA03) & - & & 4 & 0.2 & - & - \\
\hline Multiple AEDs & 1 & $<0.1$ & 36 & 1.4 & - & - \\
\hline \multirow{2}{*}{\multicolumn{7}{|c|}{$\begin{array}{l}\text { Health condition at baseline } \\
\text { Psychiatric and neurological }\end{array}$}} \\
\hline & & & & & & \\
\hline Depression & 265 & 9.0 & 199 & 7.7 & 4.6 & 0.6 \\
\hline Epilepsy & 98 & 3.3 & 425 & 16.5 & 45.2 & 11.3 \\
\hline Schizophrenia & 63 & 2.1 & 94 & 3.7 & 9.0 & 0.1 \\
\hline \multicolumn{7}{|l|}{ Hip fracture-related } \\
\hline Vision disturbances ${ }^{\mathrm{b}}$ & 1289 & 43.8 & 804 & 31.2 & 26.2 & 2.4 \\
\hline Osteoporosis ${ }^{c}$ & 683 & 23.2 & 346 & 13.4 & 25.5 & 3.6 \\
\hline Any fracture & 466 & 15.8 & 326 & 12.7 & 9.1 & 0.3 \\
\hline Head trauma & 209 & 7.1 & 223 & 8.7 & 5.8 & 2.1 \\
\hline Rheumatoid arthritis $^{d}$ & 178 & 6.0 & 88 & 3.4 & 12.4 & 3.4 \\
\hline Alcohol abuse & 78 & 2.7 & 104 & 4.0 & 7.7 & 0.7 \\
\hline \multicolumn{7}{|l|}{ Other conditions } \\
\hline Cardiovascular diseases & 1993 & 67.7 & 1591 & 61.7 & 12.4 & I.I \\
\hline Diabetes $^{\mathrm{d}}$ & 790 & 26.8 & 507 & 19.7 & 17.0 & 1.2 \\
\hline Stroke & 411 & 14.0 & 431 & 16.7 & 7.7 & I.I \\
\hline
\end{tabular}

(Continued) 
Table 2 (Continued).

\begin{tabular}{|c|c|c|c|c|c|c|}
\hline & \multicolumn{4}{|c|}{ Users $^{\mathrm{a}}$} & \multicolumn{2}{|c|}{ Standardized Mean Difference (\%) } \\
\hline & \multicolumn{2}{|c|}{ Newer AEDs ( $N=2945)$} & \multicolumn{2}{|c|}{ Older AEDs ( $N=2577)$} & & \\
\hline & $\mathbf{N}$ & $\%$ & $\mathbf{N}$ & $\%$ & Unweighted & After IPTW \\
\hline Asthma $^{d}$ & 393 & 13.3 & 229 & 8.9 & 14.2 & 1.3 \\
\hline Any cancer & 271 & 9.2 & 217 & 8.4 & 2.8 & $<0.1$ \\
\hline Chronic renal disease & 52 & 1.8 & 33 & 1.3 & 4.0 & 1.8 \\
\hline Chronic liver disease & 16 & 0.5 & 12 & 0.5 & 1.1 & 0.5 \\
\hline \multicolumn{7}{|l|}{ Drugs at baseline } \\
\hline \multicolumn{7}{|l|}{ CNS drugs } \\
\hline Benzodiazepines and related drugs & 1265 & 43.0 & 1036 & 40.2 & 5.6 & 0.7 \\
\hline Antidepressants & 1238 & 42.0 & 1040 & 40.4 & 3.4 & 3.2 \\
\hline Memantine & 984 & 33.4 & 1202 & 46.6 & 27.3 & 0.8 \\
\hline Opioids & 946 & 32.1 & 295 & 11.5 & 51.7 & 1.9 \\
\hline Antipsychotics & 708 & 24.0 & 1197 & 46.5 & 48.3 & 3.2 \\
\hline Antiparkinsonians & 178 & 6.0 & 118 & 4.6 & 6.5 & 1.7 \\
\hline \multicolumn{7}{|l|}{ Other drugs } \\
\hline Cardiovascular drugs ${ }^{f}$ & 2512 & 85.3 & 2019 & 78.4 & 18.1 & 2.8 \\
\hline PPls & 1157 & 39.3 & 644 & 25.0 & 31.0 & 3.6 \\
\hline NSAIDs & 854 & 29.0 & 358 & 13.9 & 37.4 & 4.2 \\
\hline Corticosteroids, systemic & 327 & 11.1 & 153 & 6.0 & 18.6 & 3. \\
\hline Estrogens & 315 & 10.7 & 163 & 6.3 & 15.7 & 2.6 \\
\hline Drugs for urinary frequency and incontinence & 215 & 7.3 & 104 & 4.0 & 14.2 & 3.3 \\
\hline \multicolumn{7}{|l|}{ Socioeconomic status } \\
\hline Highest & 597 & 20.3 & 666 & 25.8 & 13.3 & 0.5 \\
\hline Middle & 256 & 8.7 & 232 & 9.0 & 1.1 & 1.7 \\
\hline Lowest & 1833 & 62.2 & $|48|$ & 57.5 & 9.7 & 0.1 \\
\hline Unknown & 259 & 8.8 & 198 & 7.7 & 4.0 & I.I \\
\hline
\end{tabular}

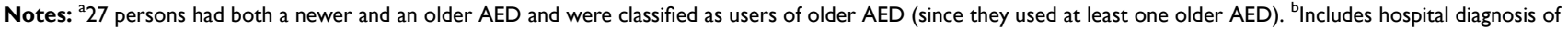
cataract, macular degeneration, and glaucoma. ${ }^{C}$ Composite of discharge diagnosis (code M80 and M8I) and dispensation data (bisphosphonates M05BA and M05BB, bone morphogenetic proteins M05BC, other drugs affecting bone structure and mineralization M05BX (eg, strontium ranelate, denosumab)). ${ }^{\mathrm{d}}$ Composite variables based on both hospitalization and special reimbursement data. ${ }^{\mathrm{e}} \mathrm{It}$ includes hypertension, heart failure, peripheral arterial disease, atrial fibrillation, coronary heart disease. ${ }^{\mathrm{f}} \mathrm{t}$ includes cardiac glycosides (COIAA), antiarrhythmics (COIB), organic nitrates (COIDA), diuretics (C03), beta blocking agents (C07), calcium channel blockers (C08), agents acting on the renin-angiotensin system (CO9), lipid modifying agents (CIOA, CIOBA), antithrombotic agents (BOIA).

Abbreviations: AD, Alzheimer's disease; AED, antiepileptic drug; ATC, anatomical therapeutic chemical; CNS, central nervous system; HF, hip fracture; IPTW, inverse probability of treatment; NSAID, nonsteroidal anti-inflammatory drugs; PPI, proton pump inhibitors.

Confounding by indication may be present in the comparison between users of AEDs and non-users, who do not have the health condition leading to pharmacotherapy, but also between newer and older AEDs. Older AEDs are mainly used to treat seizures, ${ }^{42}$ which increase the baseline risk of hip fracture. This may lead to an underestimation of the risk in users of newer AEDs if these agents are mainly used for neuropathic pain. However, newer AEDs are also used to treat neuropsychiatric symptoms (eg, hallucinations) that also increase risk of falling and fractures.

To overcome this limitation, in comparing incident users with non-users of AEDs and incident users of newer and older AEDs, we used inverse probability weighting and propensity score adjustment to balance the groups under comparison. Propensity scores were based on an extensive list of known risk factors for hip fracture, such as osteoporosis or use of psychotropic medications (eg, antidepressants, antipsychotics, benzodiazepines and related drugs), as well as of other diseases, medications and socioeconomic status to ensure that patient characteristics were captured.

Although the differences regarding these measured factors were balanced, it may be that residual confounding due to unmeasured factors persisted. Indeed, as is common in studies based on healthcare databases, direct clinical measures of certain patient characteristics, such as frailty, history of falls and degree of cognitive and functional impairment, were not available. To account for these unmeasured characteristics, 
Table 3 Age- and Sex-Adjusted Incidence Rate (IR) and Hazard Ratio (HR), with 95\% Confidence Interval (95\% Cl), of Hip Fracture (HF) According to Use of AED

\begin{tabular}{|c|c|c|c|c|c|c|c|c|c|c|c|}
\hline & \multicolumn{2}{|c|}{$\begin{array}{c}\text { Incident } \\
\text { HF (N= } \\
\text { 1028) }\end{array}$} & \multirow[t]{2}{*}{$\begin{array}{c}\text { Age- and Sex- Adjusted IR// } 00 \\
\text { Person-Years }(95 \% \mathrm{Cl})\end{array}$} & \multirow{2}{*}{\multicolumn{2}{|c|}{$\begin{array}{l}\text { Matched } H^{a} \\
\qquad(95 \% \mathrm{Cl})\end{array}$}} & \multirow{2}{*}{\multicolumn{2}{|c|}{$\begin{array}{c}\text { Propensity } \\
\text { Score Adjusted } \\
\text { HR }(95 \% \mathrm{Cl})\end{array}$}} & \multirow{2}{*}{\multicolumn{2}{|c|}{$\begin{array}{l}\text { IPTW HR } \\
(95 \% \mathrm{Cl})\end{array}$}} & \multirow{2}{*}{\multicolumn{2}{|c|}{$\begin{array}{l}\text { Stabilized IPTW } \\
\operatorname{HR}^{\mathrm{c}}(95 \% \mathrm{Cl})\end{array}$}} \\
\hline & $\mathbf{N}$ & $\%$ & & & & & & & & & \\
\hline Non-users & 673 & 65.5 & $1.8(1.6 ; 1.9)$ & \multicolumn{2}{|c|}{ Reference } & \multicolumn{2}{|c|}{ Reference } & \multicolumn{2}{|c|}{ Reference } & \multicolumn{2}{|c|}{ Reference } \\
\hline Users & 355 & 34.5 & $2.0(1.8 ; 2.2)$ & 1.24 & $(1.07 ; 1.44)$ & 1.10 & $(0.93 ; 1.29)$ & 1.17 & $(1.05 ; 1.30)$ & 1.16 & $(1.00 ; 1.36)$ \\
\hline \multicolumn{12}{|c|}{ Duration of use $\mathrm{d}^{\mathrm{d}, \mathrm{e}}$ - any AED (weeks) } \\
\hline \multicolumn{12}{|l|}{ Short $(<14)$} \\
\hline Non-users & 57 & 8.5 & $0.1(0.1 ; 0.2)$ & \multicolumn{2}{|c|}{ Reference } & \multicolumn{2}{|c|}{ Reference } & \multicolumn{2}{|c|}{ Reference } & \multicolumn{2}{|c|}{ Reference } \\
\hline Users & 112 & 31.6 & $0.6(0.5 ; 0.7)$ & 4.30 & $(3.13 ; 5.92)$ & 3.98 & $(2.84 ; 5.57)$ & 3.64 & $(2.90 ; 4.58)$ & 3.62 & $(2.65 ; 4.93)$ \\
\hline \multicolumn{12}{|c|}{ Medium $(14$ to $<64)$} \\
\hline Non-users & 161 & 23.9 & $0.4(0.3 ; 0.5)$ & \multicolumn{2}{|c|}{ Reference } & \multicolumn{2}{|c|}{ Reference } & \multicolumn{2}{|c|}{ Reference } & \multicolumn{2}{|c|}{ Reference } \\
\hline Users & 114 & 32.1 & $0.9(0.7 ; 1.1)$ & 2.23 & $(1.76 ; 2.84)$ & 1.71 & $(1.32 ; 2.21)$ & 1.74 & $(1.48 ; 2.05)$ & 1.74 & $(1.36 ; 2.22)$ \\
\hline \multicolumn{12}{|l|}{ Long ( $\geq 64)$} \\
\hline Non-users & 455 & 67.6 & $1.2(1.1 ; 1.4)$ & \multicolumn{2}{|c|}{ Reference } & \multicolumn{2}{|c|}{ Reference } & \multicolumn{2}{|c|}{ Reference } & \multicolumn{2}{|c|}{ Reference } \\
\hline Users & 129 & 36.3 & $1.7(1.4 ; 2.0)$ & 1.47 & $(1.21 ; 1.78)$ & 1.25 & (1.01; 1.54) & 1.23 & $(1.08 ; 1.40)$ & 1.24 & $(\mathrm{I} .0 \mathrm{I} ; 1.5 \mathrm{I})$ \\
\hline \multicolumn{12}{|l|}{ Type of AED } \\
\hline Older & 88 & 57.9 & $2.6(2.1 ; 3.3)$ & 1.52 & $(1.10 ; 2.10)$ & 1.10 & $(0.77 ; 1.57)$ & 1.46 & $(1.03 ; 2.08)$ & 1.37 & $(1.00 ; 1.88)$ \\
\hline Newer & 64 & 42.1 & $1.4(1.1 ; 1.9)$ & \multicolumn{2}{|c|}{ Reference } & \multicolumn{2}{|c|}{ Reference } & \multicolumn{2}{|c|}{ Reference } & & eference \\
\hline Duration of us & & & & & & & & & & & \\
\hline Short $(<14)$ & & & & & & & & & & & \\
\hline Older & 27 & 17.8 & $0.2(0,4 ; 1.0)$ & 1.88 & $(1.00 ; 3.54)$ & 1.51 & $(0.74 ; 3.05)$ & 1.27 & $(0.83 ; 1.95)$ & 1.27 & $(0.69 ; 2.33)$ \\
\hline Newer & 15 & 9.9 & $0.1(0.1 ; 0.4)$ & & eference & & eference & & eference & & eference \\
\hline Medium (14 tc & & & & & & & & & & & \\
\hline Older & 31 & 20.4 & $0.9(0.6 ; 1.3)$ & 1.60 & $(0.92 ; 2.78)$ & 1.71 & $(0.92 ; 3.18)$ & 2.00 & $(1.33 ; 3.01)$ & 2.00 & $(1.12 ; 3.56)$ \\
\hline Newer & 21 & 13.8 & $0.4(0.3 ; 0.7)$ & & eference & & eference & & eference & & eference \\
\hline Long ( $\geq 64)$ & & & & & & & & & & & \\
\hline Older & 30 & 19.7 & $1.2(0.9 ; 1.8)$ & 1.26 & $(0.75 ; 2.10)$ & 1.20 & $(0.69 ; 2.10)$ & 1.06 & $(0.75 ; 1.49)$ & 1.06 & $(0.65 ; 1.72)$ \\
\hline Newer & 28 & 18.4 & $0.9(0.6 ; 1.3)$ & & eference & & eference & & eference & & eference \\
\hline
\end{tabular}

Notes: ${ }^{a}$ Adjusted for age, sex, and time since AD diagnosis (days) by matching. ${ }^{b}$ Weighted with inverse probability of treatment weights (IPTW). ${ }^{\circ}$ Weighted with stabilized inverse probability of treatment weights (IPTW). ${ }^{\mathrm{d} C u t-o f f s}$ based on tertile distribution of duration of AED use: Ist tertile 13.9 weeks, 2 nd tertile 63.7 weeks. ${ }^{\text {eReference }}$ category for each duration of any AED use group is the non-users with same follow-up duration. ${ }^{f}$ Reference category for each duration of older AED use group is the users of newer AEDs with same duration of use.

proxy indicators have been accounted for. Specifically, we accounted for severity of $\mathrm{AD}$ by matching on time since $\mathrm{AD}$ diagnosis; we adjusted for prior fractures as indicators of (at least the most severe) falls and for alcohol abuse as a proxy of lifestyle habits. Alcohol abuse was defined based on related diagnoses and medications. This allowed to account alcohol abuse with impact on patient health, but likely not for less severe or more recent abuse. Moreover, we adjusted for socioeconomic status which is an indicator of both lifestyle habits and health status.

Exposure to AEDs was defined using dispensations that reflect medications redeemed at the pharmacy level, 


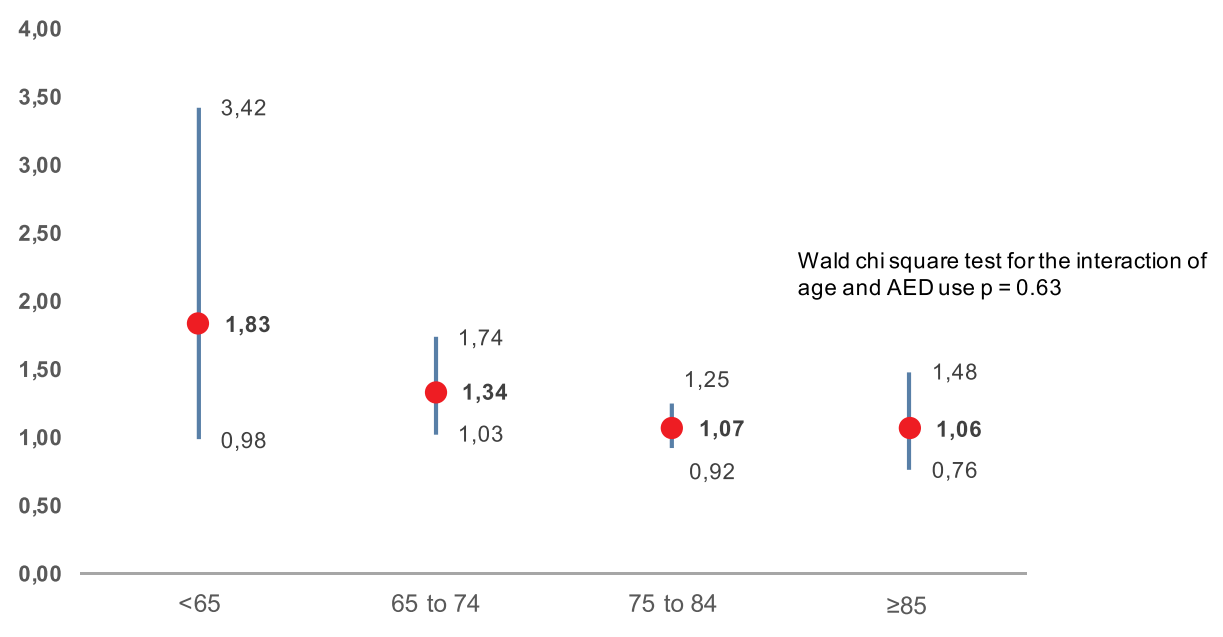

Age class (years)

Figure 2 Hazard ratio (HR) weighted for inverse probability of AED treatment, with $95 \%$ confidence interval, of hip fracture comparing incident users and non-users by age class. The following HR point estimates are in bold: I.83; I.34; I.07; 1.06.

contrary to prescriptions or information extracted from medical documentation. Thus, misclassification of the exposure should be minimal if any.

We defined the outcome as hip fracture leading to hospitalization. The completeness and accuracy of registering hip fractures is generally good in data from the Finnish Health Care Register. ${ }^{43}$ Misclassification of the outcome should thus be minimal.

\section{Conclusion}

Our results imply that AED use is associated with an increased risk of hip fracture in people with $\mathrm{AD}$. As the risk of hip fracture is higher in people with $\mathrm{AD}$ in general, prescribers need to carefully consider the risk of falling associated with antiepileptics and consider other safer options especially if prescribed to anxiety or neuropathic pain. Persons with AD treated with antiepileptics should be carefully monitored due to their increased risk of falling and fractures.

\section{Funding}

AMT is funded by Academy of Finland (grants 307232 and 295334) and acknowledges strategic funding from the University of Eastern Finland. The sponsors had no role in the design, methods, data collection, analysis and preparation of paper. This study is not industry-sponsored.

\section{Disclosure}

Dr. Heidi Taipale participated in research projects funded by Janssen-Cilag and Eli Lilly with grants paid to the institution where she was employed. Dr. Jari Tiihonen has served as a consultant to Finnish Medicines Agency (Fimea), European Medicines Agency (EMA) and JanssenCilag, received lecture fees from Janssen-Cilag, Eli Lilly, Lundbeck, and Otsuka, is a member of advisory board in Lundbeck and has participated in research projects funded by Janssen-Cilag and Eli Lilly with grants paid to the institution where he was employed. Dr. Antti Tanskanen participated in research projects funded by Janssen-Cilag and Eli Lilly with grants paid to the institution where he was employed. Dr. Sirpa Hartikainen reports personal fees from Astellas Pharma, outside the submitted work. The authors report no other conflicts of interest in this work.

\section{References}

1. Carbone LD, Johnson KC, Robbins J, et al. Antiepileptic drug use, falls, fractures, and BMD in postmenopausal women: findings from the women's health initiative (WHI). J Bone Miner Res. 2010;25 (4):873-881. doi:10.1359/jbmr.091027

2. Espinosa PS, Perez DL, Abner E, Ryan M. Association of antiepileptic drugs, vitamin D, and calcium supplementation with bone fracture occurrence in epilepsy patients. Clin Neurol Neurosurg. 2011;113 (7):548-551. doi:10.1016/j.clineuro.2011.03.011

3. Nicholas JM, Ridsdale L, Richardson MP, Grieve AP, Gulliford MC. Fracture risk with use of liver enzyme inducing antiepileptic drugs in people with active epilepsy: cohort study using the general practice research database. Seizure. 2013;22(1):37-42. doi:10.1016/j. seizure.2012.10.002

4. Souverein PC, Webb DJ, Weil JG, Van Staa TP, Egberts AC. Use of antiepileptic drugs and risk of fractures: case-control study among patients with epilepsy. Neurology. 2006;66(9):1318-1324. doi:10.1212/01.wnl.0000210503.89488.88 
5. Shen C, Chen F, Zhang Y, Guo Y, Ding M. Association between use of antiepileptic drugs and fracture risk: a systematic review and meta-analysis. Bone. 2014;64:246-253. doi:10.1016/j.bone.2014.04.018

6. Scherder EJ, Plooij B. Assessment and management of pain, with particular emphasis on central neuropathic pain, in moderate to severe dementia. Drugs Aging. 2012;29(9):701-706. doi:10.1007/ s40266-012-0001-8

7. Lanctot KL, Amatniek J, Ancoli-Israel S, et al. Neuropsychiatric signs and symptoms of Alzheimer's disease: new treatment paradigms. Alzheimers Dement. 2017;3(3):440-449. doi:10.1016/j.trci.2017.07.001

8. Seppala LJ, Petrovic M, Ryg J, et al. STOPPFall (Screening tool of older persons prescriptions in older adults with high fall risk): a Delphi study by the EuGMS task and finish group on fall-riskincreasing drugs. Age Ageing. 2020. doi:10.1093/ageing/afaa249

9. Baillon SF, Narayana U, Luxenberg JS, Clifton AV. Valproate preparations for agitation in dementia. Cochrane Database Syst Rev. 2018;10:CD003945. doi:10.1002/14651858.CD003945.pub4

10. Liu CS, Ruthirakuhan M, Chau SA, Herrmann N, Carvalho AF, Lanctot KL. pharmacological management of agitation and aggression in Alzheimer's disease: a review of current and novel treatments. Curr Alzheimer Res. 2016;13(10):1134-1144. doi:10.2174/ 1567205013666160502122933

11. Gallagher D, Herrmann N. Antiepileptic drugs for the treatment of agitation and aggression in dementia: do they have a place in therapy? Drugs. 2014;74(15):1747-1755. doi:10.1007/s40265-014-0293-6

12. Dolder CR, Nealy KL. The efficacy and safety of newer anticonvulsants in patients with dementia. Drugs Aging. 2012;29(8):627-637. doi:10.1007/BF03262279

13. Watt JA, Goodarzi Z, Veroniki AA, et al. Safety of pharmacologic interventions for neuropsychiatric symptoms in dementia: a systematic review and network meta-analysis. BMC Geriatr. 2020;20(1):212. doi:10.1186/s12877-020-01607-7

14. Tolppanen AM, Taipale H, Koponen M, et al. Screening approach for identifying candidate drugs and drug-drug interactions related to hip fracture risk in persons with Alzheimer disease. Pharmacoepidemiol Drug Saf. 2017;26(8):875-889. doi:10.1002/pds.4232

15. Sarycheva T, Taipale H, Lavikainen $P$, et al. Incidence and prevalence of antiepileptic medication use in community-dwelling persons with and without Alzheimer's disease. J Alzheimers Dis. 2018;66 (1):387-395. doi:10.3233/JAD-180594

16. Tolppanen A-M, Lavikainen P, Soininen H, Hartikainen S. Incident hip fractures among community dwelling persons with alzheimer's disease in a finnish nationwide register-based cohort. PLoS One. 2013;8(3):e59124. doi:10.1371/journal.pone.0059124

17. Kanis JA, Oden A, McCloskey EV, Johansson H, Wahl DA, Cooper C. A systematic review of hip fracture incidence and probability of fracture worldwide. Osteoporos Int. 2012;23(9):2239-2256. doi:10.1007/s00198-012-1964-3

18. Bliuc D, Nguyen ND, Milch VE, Nguyen TV, Eisman JA. Mortality risk associated with low-trauma osteoporotic fracture and subsequent fracture in men and women. JAMA. 2009;301(5):513-521. doi:10.1001/jama.2009.50

19. Klop C, van Staa TP, Cooper C, Harvey NC, de Vries F. The epidemiology of mortality after fracture in England: variation by age, sex, time, geographic location, and ethnicity. Osteoporos Int. 2017;28(1):161-168. doi:10.1007/s00198-016-3787-0

20. Katsoulis M, Benetou V, Karapetyan T, et al. Excess mortality after hip fracture in elderly persons from Europe and the USA: the CHANCES project. J Intern Med. 2017;281(3):300-310. doi:10.1111/joim.12586

21. Dahl C, Holvik K, Meyer HE, et al. Increased mortality in hip fracture patients living alone: a NOREPOS Study. J Bone Miner Res. 2021;36(3):480-488. doi:10.1002/jbmr.4212

22. Patel R, Bhimjiyani A, Ben-Shlomo Y, Gregson CL. Social deprivation predicts adverse health outcomes after hospital admission with hip fracture in England. Osteoporos Int. 2021. doi:10.1007/s00198020-05768-4
23. Tajeu GS, Delzell E, Smith W, et al. Death, debility, and destitution following hip fracture. J Gerontol a Biol Sci Med Sci. 2014;69 (3):346-353. doi:10.1093/gerona/glt105

24. Tang VL, Sudore R, Cenzer IS, et al. Rates of recovery to pre-fracture function in older persons with hip fracture: an observational study. J Gen Intern Med. 2017;32(2):153-158. doi:10.1007/ s11606-016-3848-2

25. Bleibler F, Rapp K, Jaensch A, Becker C, Konig HH. Expected lifetime numbers and costs of fractures in postmenopausal women with and without osteoporosis in Germany: a discrete event simulation model. BMC Health Serv Res. 2014;14(1):284. doi:10.1186/ 1472-6963-14-284

26. Lambrelli D, Burge R, Raluy-Callado M, Chen SY, Wu N, Schoenfeld MJ. Retrospective database study to assess the economic impact of hip fracture in the United Kingdom. J Med Econ. 2014;17 (11):817-825. doi:10.3111/13696998.2014.959588

27. Leal J, Gray AM, Prieto-Alhambra D, et al. Impact of hip fracture on hospital care costs: a population-based study. Osteoporos Int. 2016;27(2):549-558. doi:10.1007/s00198-015-3277-9

28. Tarride J, Adachi JD, Brown JP, Schemitsch E, Slatkovska L, Burke N. Incremental costs of fragility fractures: a population-based matched-cohort study from Ontario, Canada. Osteoporos Int. 2021. doi:10.1007/s00198-021-05877-8

29. Tolppanen AM, Taipale H, Koponen M, et al. Cohort profile: the Finnish medication and Alzheimer's disease (MEDALZ) study. BMJ Open. 2016;6(7):e012100. doi:10.1136/bmjopen-2016-012100

30. McKhann G, Drachman D, Folstein M, Katzman R, Price D, Stadlan EM. Clinical diagnosis of Alzheimer's disease. Report of the NINCDS-ADRDA Work Group* under the auspices of Department of health and human services task force on Alzheimer's disease. Neurology. 1984;34(7):939. doi:10.1212/WNL.34.7.939

31. Bell CC. DSM-IV: diagnostic and statistical manual of mental disorders. JAMA. 1994;272(10):828-829. doi:10.1001/ jama.1994.03520100096046

32. Ray WA. Evaluating medication effects outside of clinical trials: new-user designs. Am J Epidemiol. 2003;158(9):915-920. doi:10.1093/aje/kwg231

33. Tanskanen A, Taipale H, Koponen M, et al. From prescription drug purchases to drug use periods - a second generation method (PRE2DUP). BMC Med Inform Decis Mak. 2015;15(1):21. doi:10.1186/s12911-015-0140-z

34. Taipale H, Tanskanen A, Koponen M, Tolppanen AM, Tiihonen J, Hartikainen S. Agreement between PRE2DUP register data modeling method and comprehensive drug use interview among older persons. Clin Epidemiol. 2016;8:363-371. doi:10.2147/CLEP.S116160

35. Austin PC. An introduction to propensity score methods for reducing the effects of confounding in observational studies. Multivariate Behav Res. 2011;46(3):399-424. doi:10.1080/00273171.2011.568786

36. Austin PC, Stuart EA. Moving towards best practice when using inverse probability of treatment weighting (IPTW) using the propensity score to estimate causal treatment effects in observational studies. Stat Med. 2015;34(28):3661-3679. doi:10.1002/sim.6607

37. Austin PC. Goodness-of-fit diagnostics for the propensity score model when estimating treatment effects using covariate adjustment with the propensity score. Pharmacoepidemiol Drug Saf. 2008;17 (12):1202-1217. doi:10.1002/pds.1673

38. Vajda FJ, Eadie MJ. The clinical pharmacology of traditional antiepileptic drugs. Epileptic Disord. 2014;16(4):395-408. doi:10.1684/ epd.2014.0704

39. Clement ND, Court-Brown CM. Elderly pelvic fractures: the incidence is increasing and patient demographics can be used to predict the outcome. Eur J Orthop Surg Traumatol. 2014;24(8):1431-1437. doi:10.1007/s00590-014-1439-7

40. Court-Brown CM, McQueen MM. Global Forum: fractures in the Elderly. J Bone Joint Surg Am. 2016;98(9):e36. doi:10.2106/ JBJS.15.00793 
41. Bhandari SK, Adams AL, Li BH, et al. Sub-acute more than chronic hyponatremia is associated with serious falls and hip fractures. Intern Med J. 2019.

42. Vossel KA, Tartaglia MC, Nygaard HB, Zeman AZ, Miller BL. Epileptic activity in Alzheimer's disease: causes and clinical relevance. Lancet Neurol. 2017;16(4):311-322. doi:10.1016/S14744422(17)30044-3
43. Sund R, Nurmi-Luthje I, Luthje P, Tanninen S, Narinen A, Keskimaki I. Comparing properties of audit data and routinely collected register data in case of performance assessment of hip fracture treatment in Finland. Methods Inf Med. 2007;46(05):558-566. doi:10.1160/ME0382

\section{Publish your work in this journal}

Clinical Epidemiology is an international, peer-reviewed, open access, online journal focusing on disease and drug epidemiology, identification of risk factors and screening procedures to develop optimal preventative initiatives and programs. Specific topics include: diagnosis, prognosis, treatment, screening, prevention, risk factor modification,

Submit your manuscript here: https://www.dovepress.com/clinical-epidemiology-journal systematic reviews, risk \& safety of medical interventions, epidemiology \& biostatistical methods, and evaluation of guidelines, translational medicine, health policies \& economic evaluations. The manuscript management system is completely online and includes a very quick and fair peer-review system, which is all easy to use. 